\title{
Book Flipping And Scanning Machine Review
}

\author{
Priyanka Bhanudas Deshmukh \\ ME E\&TC(VLSI \& EMBEDED SYSTEM) \\ G.H.RAISONI COEM \\ AHMEDNAGAR INDIA
}

\begin{abstract}
With the advent of digital technology, it is to access a huge number of documents from virtually any location. Unfortunately, the world's libraries are filled with books and other bound documents that are not available except through direct physical access to the material. Hence, much of the wisdom and knowledge of the past remains unused. Worse, there is a possibility that many older documents will be discarded due to the cost of storage and maintenance, so their will be loss to our civilization. Although paper documents can be preserved by imaging and digitization, manual book scanning is expensive and tedious. As a result, lesser-used documents tend to have a lower scanning priority. Our future generations will undoubtedly find that many of the lost documents would been crucial to their research. Hence scanning of books and documents is vital as well as necessary.
\end{abstract}

Keywords— book scanning,flipping,research work,matlab,arm7

\section{INTRODUCTION}

Portable tablet computers and smart phones are widely used, and different services depend on digital and sharing of a mass amount of data are being developed. Along with this technology and trend, particularly in the form of books, is fetching demand and book digitization is developing fast in whole world. High-speed book digitization is vital technology which put a firm impact in many fields, like storing documents. A vital technology that made important growth under this situation, called Book Flipping Scanning, has been introduced. This is a new method of scanning books in which all pages of a book are captured while pages are continuously flipped automatically through the page turner without stopping at each page. Many systems introducing this concept are introduced and developed to reach the different requirements in different markets, a high-speed camera system and a portable system using a single camera This paper introduce a new book-page turner machine that provides three demands - high speed, accuracy, and automatic operation - so that an innovative high-speed book digitization technology based on Book Flipping Scanning.

\section{RESEARCH WORK ON RELATED TECHNOLOGY.}

1. The kirtas APT Book scan 1200 has developed fully automated device that scans and digitize books at rate of 1200 pages per hour. The IEEE control systems magazine spoke to Dr. Belkhir for understanding the operation of kirtas technology [1].

2. Low over head Manipulation of bound book pages this article describes new method for one sided, nonprehensile paper manipulation problem in which we use polydimethylsiloxane to create mechanical bond between the paper and robot manipulator. The robot has scan pages 10720 at $0.056 \%$ error rate [2].

3. Automatic page turner machine for high speed book digitization. This article achieved almost $100 \%$ success rate for turning pages at rate of 300 pages per min [3].

\section{BASIC PRINCIPAL OF ABOVE MENTIONED TECHNOLOGY.}

1.Kirtas technology

Page-handling technology invented for the Kirtas APT BookScan 1200. The V-shaped SmartCradle uses independently moving plates to precisely maintain the top pages of the book in the focal plane of the camera, while the Sure- Turn robotic arm uses a vacuum pickup head, edge detection sensors, and puffs of air to reliably turn pages whose texture ranges from very thin paper to photographic paper.[1]

2.Low overhead manipulation of book bound pages

The flexibility of the paper plus limitations imposed by the spine can be actively exploited in order to separate a book leaf from following leaves. Because a single side of each book leaf is highly exposed at a time, nonprehensile methods for paper manipulation are particularly well suited to this problem in contrast to more conventional grasp-based techniques that would require access to both sides of the paper.[2]

3.Automatic page turner machine for high speed book digitization .

This paper describes a new book-page turner machine that meeting these three demands high speed, accuracy, 
and automatic operation in order to establish an innovative high-speed book digitization technology based on Book Flipping Scanning. in order to specialize the machine for book digitization, we adopt a new configuration that is completely different from conventional systems, in which no handling device is placed on the paper surface, so that the entire document image can be captured.[3]

\section{OPERATION AND RESPECTIVE MODELS.}

1.Real page turner.

The SureTurn robotic arm incorporates a vacuum pickup head and accommodates book sizes ranging from $5 \_\times 8$ _ to $10 \_\times 13$. The system uses a sensor that detects the page edge during operation, and automatically maintains the pickup head at a fixed distance from the page edge. This same sensor is also used to detect page drops, multi feeds, and loose pages, helping to ensure gentle, reliable, and error-free page turning. The Vshaped Smart Cradle is comprised of two independently moving plates that dynamically and precisely maintain the top pages of the book in the focal plane of the camera. The heights of the left and right pages are continuously monitored by a set of optical sensors.

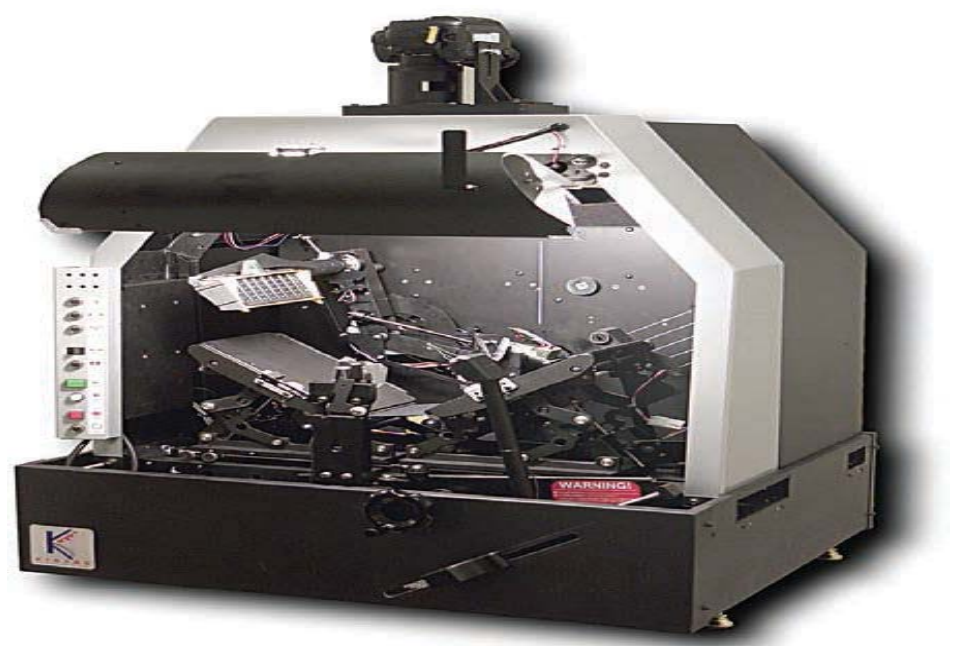

Fig 1 Real page turner.

\section{Low overhead manipulation of bound book pages.}

We can build a topological map that runs from cover to cover, which designates the ordering of each page and robot arm.

This can be conceptualized by creating a semi-circular arc that spans from cover to cover and is centered at the spine. When the arc intersects a page or robot arm, a node is created in a connected graph. This produces a graph that describes the interleaving of the robot's manipulators and the book's leaves. The robot requires five arms that interpose themselves between different leaves and/or other robot arms throughout the cycle. F is used to manipulate the surface of R0 to facilitate the isolation of R0 from R1.

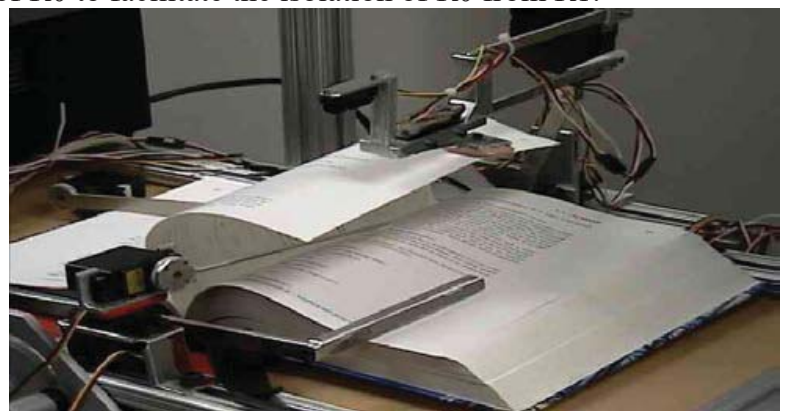

Fig 2 low overhead manipulation model. Speed digitization

3.Automatic page turner machine for high speed book digitization.

This machine manipulates a planar surface formed by the edges of the pages at the opposite side from the spine when 
the book is curved. We call this part the edge face in this paper. At this edge face, we confirmed that the pages are overlapped and aligned at regular intervals, and their ends are approximately parallel in a local region. Therefore, by moving a manipulator perpendicular to the ends of the pages while maintaining the edge face shape, when each page is set free, it can rise due to an elastic force caused by the curvature. By blowing air into the space formed between the rising page and the next page below it, the paper will be turned to the opposite side.

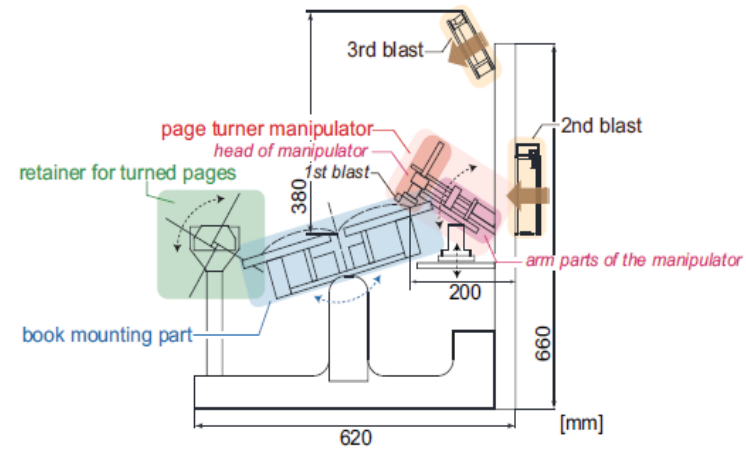

Fig 3 Automatic page turner machine.

\section{CONCLUSION}

Thus we can conclude that the low manipulation machine can provide low cost low overhead solutions to presenting every side of a book leaves properly .thus we can view every side of book leaves properly. The automatic book scanning machine can give $100 \%$ error free digitization of book.

But in future development of the machine which can give $100 \%$ digitization at lower cost is possible. We can thus bring out the prototype which can cost much leaser than photocopy machine and scanning machine in market.

\section{REFERENCES}

[1] A Real page turner IEEE control systems magazine April 2004.

[2] Josh Young ,Illah R.Nourbakhsh."Low overhead Manipulation of Book Bound Pages" IEEE 2004 international conference on Robotics and Automation.

[3] Yoshihro Watanabe, Miho Tamer, Masahiro Yamada, M Ishikawa "Automatic page turner machine for high speed book digitization"IEEE/RSJ 2013.

[4] T. Nakashima, Y. Watanabea, T. Komuro, and M. Ishikawa, "Book flipping scanning," in 22nd Symposium on User Interface Software and Technology, 2009, pp. 79-80.

[5] Y. Watanabe, K. Itoyama, M. Yamada, and M. Ishikawa, "Digitization of deformed documents using a high-speed multi-camera array," in The 11th Asian Conference on Computer Vision, 2012, pp. 394-407.

[6] H. Shibayama, Y. Watanabe, and M. Ishikawa, "Reconstruction of 3D surface and restoration of flat document image from monocular image sequence," in The 11th Asian Conference on Computer Vision, 2012, pp. 350-364.

[7] H. Cheng, H. Ikeda, and K. Yoshida, "Numerical analysis on paper sheet separation using the overlap separation mechanism," Journal of Advanced Mechanical Design, Systems, and Manufacturing, vol. 4, no. 1, pp. 249-256, 2010.

[8] W. M. Cho, "Paper feed device for duplex printing apparatus," Patent EP0 919880 B1, Aug. 14, 2002.

[9] E. Abueg, J. Coughlin, N. Hanumara, O. Koenig, A. Tillinghast, and T. Malaghan, "Implementation of a re-usable adhesive in a novel automated page-turner," in IEEE Bioengineering Conference, 2004, pp. 208-209. 